Pacific

Journal of

Mathematics

WEB BASES FOR sl(3) ARE NOT DUAL CANONICAL

Mikhail Khovanov and Greg KuperberG

Volume 188 No. 1

March 1999 


\title{
WEB BASES FOR sl(3) ARE NOT DUAL CANONICAL
}

\author{
Mikhail Khovanov and Greg Kuperberg
}

We compare two natural bases for the invariant space of a tensor product of irreducible representations of $A 2$, or $\mathrm{sl}(3)$. One basis is the web basis, defined from a skein theory called the combinatorial $A 2$ spider. The other basis is the dual canonical basis, the dual of the basis defined by Lusztig and Kashiwara. For $\mathrm{sl}(2)$ or $A 1$, the web bases have been discovered many times and were recently shown to be dual canonical by Frenkel and Khovanov.

We prove that for $\operatorname{sl}(3)$, the two bases eventually diverge even though they agree in many small cases. The first disagreement comes in the invariant space $\operatorname{Inv}\left(\left(V^{+} \otimes V^{+} \otimes V^{-}\right.\right.$ $\left.\left.\otimes V^{-}\right)^{\otimes 3}\right)$, where $V^{+}$and $V^{-}$are the two 3-dimensional representations of $\mathrm{sl}(3)$; if the tensor factors are listed in the indicated order, only 511 of the 512 invariant basis vectors coincide.

\section{Introduction.}

Given a simple Lie algebra $\mathfrak{g}$ over $\mathbb{C}$ and a finite list of finite-dimensional, irreducible representations $V_{1}, V_{2}, \ldots, V_{n}$, one can study different bases of the tensor product representation

$$
V_{1} \otimes V_{2} \otimes \ldots \otimes V_{n}
$$

or its invariant space

$$
\operatorname{Inv}\left(V_{1} \otimes V_{2} \otimes \ldots \otimes V_{n}\right) .
$$

The quantum group $U_{q}(\mathfrak{g})$ has representations and vector spaces of invariants which generalize these, and one can also study their bases, with or without the intention of specializing to $q=1$. (For simplicity, we will usually consider $U_{q}(\mathfrak{g})$ as an algebra over $\mathbb{C}\left(q^{1 / 2}\right)$, and we will only occassionally mention $\mathbb{Z}\left[q^{ \pm 1 / 2}\right]$ as a ground ring.) Lusztig's remarkable canonical bases [6], which are the same as Kashiwara's crystal bases [2], extend to bases of these spaces and have many important properties.

When $\mathfrak{g}=\operatorname{sl}(2)$, the Temperley-Lieb category $[\mathbf{3}, \mathbf{1}]$ gives another set of bases for the invariant spaces. It was recently established that these bases are dual canonical, i.e., dual in the sense of linear algebra to canonical bases 
[1]. The Temperley-Lieb category gives a particularly explicit, simple, and useful definition of the dual canonical bases of invariants (dual canonical invariants) which establishes further natural properties of these bases.

Reference [5] defines generalizations of the Temperley-Lieb category to the three rank two Lie algebras $A_{2} \cong \operatorname{sl}(3), B_{2} \cong \operatorname{sp}(4) \cong \operatorname{so}(5)$, and $G_{2}$. These generalizations are called combinatorial rank two spiders. The bases they yield are called web bases and their individual basis vectors are called webs. One may conjecture that these bases are also dual canonical. As evidence for the conjecture, consider the following properties which the $A_{2}$ web bases share with dual canonical invariants:

1: Let $V_{1}, V_{2}, \ldots, V_{n}$ be arbitrary irreducible representations of $U_{q}(\operatorname{sl}(3))$. Then there is a natural cyclic permutation operator

$$
\operatorname{Inv}\left(V_{1} \otimes V_{2} \otimes \ldots \otimes V_{n}\right) \rightarrow \operatorname{Inv}\left(V_{2} \otimes V_{3} \otimes \ldots \otimes V_{n} \otimes V_{1}\right),
$$

and it sends basis webs to other basis webs. (Reference [6], Prop. 28.2.4, establishes this property for dual canonical invariants.)

2: The tensor product of two basis webs is a basis web. (For dual canonical invariants, this is a corollary of Theorem 3.)

3: If two adjacent tensor factors of a basis web are dual 3-dimensional representations, then contracting them produces a linear combination of basis webs with coefficients in $\mathbb{N}\left[-q^{1 / 2},-q^{-1 / 2}\right]$. (Here $\mathbb{N}$ means the non-negative integers; the property is conjectural for dual canonical bases.)

4: Let $V$ and $V^{\prime}$ be tensor products of arbitrary irreducible representations, and let $V(\lambda)$ be the irreducible representation of highest weight $\lambda$. Then $\operatorname{Inv}(V \otimes B)$ decomposes as

$$
\operatorname{Inv}(A \otimes B) \cong \bigoplus_{\lambda} \operatorname{Inv}(A \otimes V(\lambda)) \otimes \operatorname{Inv}\left(V\left(\lambda^{*}\right) \otimes B\right) .
$$

This decomposition induces a grading by $\lambda$, which leads to two filtrations by the usual partial ordering on dominant weights. The web basis refines the ascending filtration.

5: The web bases are dual canonical in small cases.

In this paper, we will disprove the conjecture. Let $V^{+}$be the defining 3 -dimensional representation of $\operatorname{sl}(3)$ and let $V^{-}$be the dual representation, and let $V^{+}$and $V^{-}$also denote the corresponding representations of $U_{q}(\operatorname{sl}(3))$. Then:

Theorem 1. Every basis web in

$$
\operatorname{Inv}\left(V_{1} \otimes V_{2} \otimes \ldots \otimes V_{n}\right),
$$

where each $V_{i}$ is either $V^{+}$or $V^{-}$, is dual canonical when $n \leq 12$, except for a single basis web in

$$
\operatorname{Inv}\left(\left(V^{+} \otimes V^{+} \otimes V^{-} \otimes V^{-}\right)^{\otimes 3}\right)
$$


and its counterparts given by cyclic permutation of tensor factors.

To see the extent of early agreement between the two kinds of bases, note that there are 35 permutations of six tensor factors of $V^{+}$and six tensor factors of $V^{-}$which are inequivalent under sign flip, reversal of order, and cyclic permutation. Each permutation yields a 513-dimensional vector space of invariants. All 513 basis webs are dual canonical unless the $V^{+}$'s and the $V^{-}$'s are in the arrangement stated in the theorem, in which case 512 of them are. However, the fraction of basis webs that are dual canonical must go to 0 exponentially as $n \rightarrow \infty$.

In comparing the two types of bases, we will often refer to the book by Lusztig [6]. The results cited there are stated in terms of canonical bases, but they can be translated to statements about dual canonical bases.

\subsection{Acknowledgements.}

The authors would like to thank Igor Frenkel for his attention to this work.

\section{The quantum group $U_{q}(\mathrm{sl}(3))$.}

The quantum group $U_{q}(\operatorname{sl}(3))$ is an associative algebra over $\mathbb{C}\left(q^{1 / 2}\right)$, where $q^{1 / 2}$ is an indeterminate, with generators $E_{i}, F_{i}, K_{i}$, and $K_{i}^{-1}$ for $i=1,2$, and the following relations:

$$
\begin{gathered}
K_{i} K_{i}^{-1}=K_{i}^{-1} K_{i}=1 \\
K_{i} K_{j}=K_{j} K_{i} \\
K_{i} E_{j}=q^{a_{i j} / 2} E_{j} K_{i} \\
K_{i} F_{j}=q^{-a_{i j} / 2} F_{j} K_{i} \\
E_{i} F_{i}-F_{i} E_{i}=\delta_{i j} \frac{K_{i}-K_{i}^{-1}}{q-q^{-1}} \\
E_{i}^{2} E_{j}-[2] E_{i} E_{j} E_{i}+E_{j} E_{i}^{2}=0 \quad i \neq j \\
F_{i}^{2} F_{j}-[2] F_{i} F_{j} F_{i}+F_{j} F_{i}^{2}=0 \quad i \neq j .
\end{gathered}
$$

Here $\delta_{i j}$ is 1 when $i=j$ and 0 when $i \neq j$, while $a_{i j}=3 \delta_{i j}-1$ is the Cartan matrix of $\operatorname{sl}(3)$. The quantity $[n]$ is a quantum integer, defined by

$$
[n]=\frac{q^{n / 2}-q^{-n / 2}}{q^{1 / 2}-q^{-1 / 2}} .
$$

After clearing denominators in the relations, one obtains a Hopf algebra over $\mathbb{Z}\left[q^{1 / 2}, q^{-1 / 2}\right]$. For convenience, let $v=-q^{1 / 2}$. (Our $v$ is the negative of the $v$ in Reference [6].)

The algebra $U_{q}(\operatorname{sl}(3))$ is a Hopf algebra with a certain standard coproduct $\Delta$. In this paper, we will use a second coproduct $\bar{\Delta}$ which is more appropriate for dual canonical bases. This coproduct takes the following values on 
generators:

$$
\begin{aligned}
\bar{\Delta}\left(K_{i}^{ \pm 1}\right) & =K_{i}^{ \pm 1} \otimes K_{i}^{ \pm 1} \\
\bar{\Delta}\left(E_{i}\right) & =E_{i} \otimes 1+K_{i}^{-1} \otimes E_{i} \\
\bar{\Delta}\left(F_{i}\right) & =F_{i} \otimes K_{i}+1 \otimes F_{i} .
\end{aligned}
$$

We use this coproduct to understand $V \otimes V^{\prime}$ as a representation of $U_{q}(\mathrm{sl}(3))$ if $V$ and $V^{\prime}$ are themselves representations. Also, in any representation, we will say that $e$ is an invariant vector if $X e=\epsilon(X) e$, where $\epsilon$ is a homomorphism from $U_{q}(\operatorname{sl}(3))$ to $\mathbb{C}(v)$ given on generators by

$$
\begin{aligned}
\epsilon\left(E_{i}\right) & =\epsilon\left(F_{i}\right)=0 \\
\epsilon\left(K_{i}\right) & =1 .
\end{aligned}
$$

The vector space of all invariants of $V$ is denoted $\operatorname{Inv}(V)$.

The two irreducible representations of the quantum group $U_{q}(\operatorname{sl}(3))$ that we will study are the 3-dimensional representations $V^{+}$and $V^{-}$. We choose a basis $e_{-1}^{ \pm}, e_{0}^{ \pm}, e_{1}^{ \pm}$of $V^{ \pm}$; the action of $U_{q}(\operatorname{sl}(3))$ on $V^{+}$is given by:

$$
\begin{aligned}
& K_{1}\left(e_{1}^{+}\right)=q^{1 / 2} e_{1}^{+} \quad K_{1}\left(e_{0}^{+}\right)=q^{-1 / 2} e_{0}^{+} \quad K_{1}\left(e_{-1}^{+}\right)=e_{-1}^{+} \\
& K_{2}\left(e_{1}^{+}\right)=e_{1}^{+} \quad K_{2}\left(e_{0}^{+}\right)=q^{1 / 2} e_{0}^{+} \quad K_{2}\left(e_{-1}^{+}\right)=q^{-1 / 2} e_{-1}^{+} \\
& E_{1}\left(e_{0}^{+}\right)=e_{1}^{+} \quad F_{1}\left(e_{1}^{+}\right)=e_{0}^{+} \\
& E_{2}\left(e_{-1}^{+}\right)=e_{0}^{+} \quad F_{2}\left(e_{0}^{+}\right)=e_{-1}^{+}
\end{aligned}
$$

and all other combinations such as $E_{1}\left(v_{1}\right)$ are 0 . Similarly, the action on $V^{-}$is given by:

$$
\begin{aligned}
& K_{1}\left(e_{1}^{-}\right)=e_{1}^{-} \quad K_{1}\left(e_{0}^{-}\right)=q^{1 / 2} e_{0}^{-} \quad K_{1}\left(e_{-1}^{-}\right)=q^{-1 / 2} e_{-1}^{-} \\
& K_{2}\left(e_{1}^{-}\right)=q^{1 / 2} e_{1}^{-} \quad K_{2}\left(e_{0}^{-}\right)=q^{-1 / 2} e_{0}^{-} \quad K_{2}\left(e_{-1}^{-}\right)=e_{-1}^{-} \\
& E_{1}\left(e_{-1}^{-}\right)=e_{0}^{-} \quad F_{1}\left(e_{0}^{-}\right)=e_{-1}^{-} \\
& E_{2}\left(e_{0}^{-}\right)=e_{1}^{-} \quad F_{2}\left(e_{1}^{-}\right)=e_{0}^{-}
\end{aligned}
$$


and all other combinations are 0 . These actions are summarized by the weight diagrams of $V^{+}$and $V^{-}$:
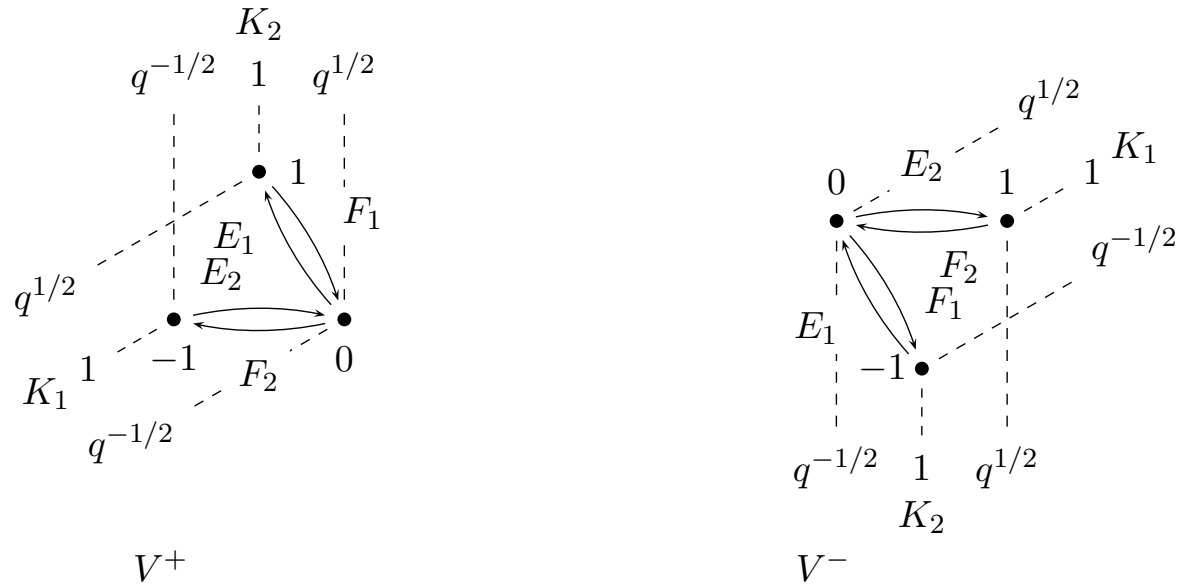

These bases for $V^{+}$and $V^{-}$are dual canonical because they satisfy the conditions of bases at $\infty[6,20.1 .1]$.

\section{The combinatorial $A_{2}$ spider.}

Strictly speaking, the combinatorial $A_{2}$ spider is an abstract tensor category given by generators and relations which is known to be isomorphic to the category of intertwiners of $U_{q}(\operatorname{sl}(3))$ [5]. However, in this paper, this isomorphism will be implicit and we will instead describe vectors of $U_{q}(\operatorname{sl}(3))$, called webs, which are associated to it.

Following Reshetikhin and Turaev [8], we denote invariants and equivariants in the representation category of $U_{q}(\mathrm{sl}(3))$ by means of planar graphs. If $V$ is a tensor product of $V^{ \pm}$'s, an element of $\operatorname{Inv}(V)$ may be denoted by some graph with oriented edges, with vertices labelled by invariant tensors, and with a univalent vertex for each tensor factor. For each factor of $V^{+}$, the edge is oriented towards the corresponding vertex, and for each factor of $V^{-}$, the edge is oriented away:

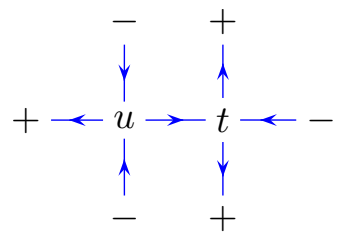

As in this example, we abbreviate $V^{+}$and $V^{-}$by their signs. Sometimes the signs or the orientations or both will be omitted in cases where they are irrelevant or clear from context. 
If $V$ and $V^{\prime}$ are two different tensor products, the equivalence $\operatorname{Hom}\left(V, V^{\prime}\right)$ $\cong \operatorname{Inv}\left(V^{*} \otimes V^{\prime}\right)$ will also be important. For example, a graph such as:

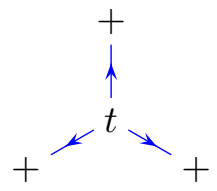

might denote an element of $\operatorname{Hom}\left(V^{-}, V^{+} \otimes V^{+}\right)$just as well as an element of $\operatorname{Inv}\left(\left(V^{+}\right)^{\otimes 3}\right)$. Using this equivalence, compositions of homomorphisms are in general denoted by concatenation and tensor products of homomorphisms (or invariants) are denoted by juxtaposition, or disjoint union.

The combinatorial $A_{2}$ webs can be constructed from four elementary invariants and the operations of tensor product and contraction. The four invariants are

$$
\begin{aligned}
b^{+-}= & e_{1}^{+} \otimes e_{-1}^{-}+v^{-1} e_{0}^{+} \otimes e_{0}^{-}+v^{-2} e_{-1}^{+} \otimes e_{1}^{-} \\
b^{-+}= & e_{1}^{-} \otimes e_{-1}^{+}+v^{-1} e_{0}^{-} \otimes e_{0}^{+}+v^{-2} e_{-1}^{-} \otimes e_{1}^{+} \\
t^{---}= & e_{1}^{-} \otimes e_{0}^{-} \otimes e_{-1}^{-}+v^{-1} e_{0}^{-} \otimes e_{1}^{-} \otimes e_{-1}^{-}+v^{-1} e_{1}^{-} \otimes e_{-1}^{-} \otimes e_{0}^{-} \\
& \quad+v^{-2} e_{0}^{-} \otimes e_{-1}^{-} \otimes e_{1}^{-}+v^{-2} e_{-1}^{-} \otimes e_{1}^{-} \otimes e_{0}^{-}+v^{-3} e_{-1}^{-} \otimes e_{0}^{-} \otimes e_{1}^{-} \\
t^{+++}= & e_{1}^{+} \otimes e_{0}^{+} \otimes e_{-1}^{+}+v^{-1} e_{0}^{+} \otimes e_{1}^{+} \otimes e_{-1}^{+}+v^{-1} e_{1}^{+} \otimes e_{-1}^{+} \otimes e_{0}^{+} \\
& \quad+v^{-2} e_{0}^{+} \otimes e_{-1}^{+} \otimes e_{1}^{+}+v^{-2} e_{-1}^{+} \otimes e_{1}^{+} \otimes e_{0}^{+}+v^{-3} e_{-1}^{+} \otimes e_{0}^{+} \otimes e_{1}^{+} .
\end{aligned}
$$

The contraction operations are defined using the equivariant pairings $\sigma_{+-}$: $V^{+} \otimes V^{-} \rightarrow \mathbb{C}(q)$ and $\sigma_{-+}: V^{-} \otimes V^{+} \rightarrow \mathbb{C}(q)$, which are given by

$$
\begin{aligned}
\sigma_{+-}\left(e_{-1}^{+} \otimes e_{1}^{-}\right) & =\sigma_{-+}\left(e_{-1}^{-} \otimes e_{1}^{+}\right)=1 \\
\sigma_{+-}\left(e_{0}^{+} \otimes e_{0}^{-}\right) & =\sigma_{-+}\left(e_{0}^{-} \otimes e_{0}^{+}\right)=v \\
\sigma_{+-}\left(e_{1}^{+} \otimes e_{-1}^{-}\right) & =\sigma_{-+}\left(e_{1}^{-} \otimes e_{-1}^{+}\right)=v^{2}
\end{aligned}
$$

and all other values on basis vectors are 0 . Since the $\sigma$ 's are equivariant, they induce contractions

$$
\operatorname{Inv}\left(V \otimes V^{ \pm} \otimes V^{\mp} \otimes V^{\prime}\right) \rightarrow \operatorname{Inv}\left(V \otimes V^{\prime}\right)
$$

for arbitrary tensor products $V$ and $V^{\prime}$.

The planar graphs corresponding to the $b$ 's and $t$ 's are:

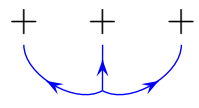

$t^{+++}$

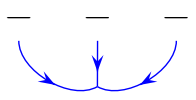

$t^{---}$

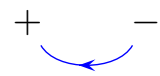

$b^{+-}$

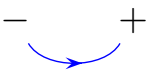

$b^{-+}$ 
while those corresponding to the $\sigma$ 's are

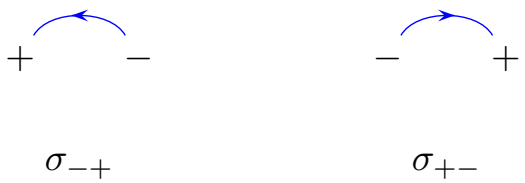

If the $b$ 's and $t$ 's are understood as equivariant homomorphisms from the ground field $\mathbb{C}(v)$ to the corresponding invariant spaces, they and the $\sigma$ 's can be composed to form planar graphs. For example, the graph

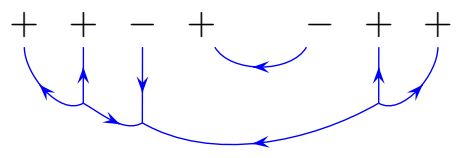

denotes the tensor

$$
\left(I \otimes I \otimes \sigma_{+-} \otimes I \otimes b^{+-} \otimes \sigma_{-+} \otimes I \otimes I\right) \circ\left(t^{+++} \otimes t^{---} \otimes t^{+++}\right) .
$$

Given the identities

$$
\begin{aligned}
& \left(I \otimes \sigma_{+-}\right) \circ\left(b^{-+} \otimes I\right)=\left(\sigma_{-+} \otimes I\right) \circ\left(I \otimes b^{+-}\right)=I \\
& \left(I \otimes \sigma_{-+}\right) \circ\left(b^{+-} \otimes I\right)=\left(\sigma_{+-} \otimes I\right) \circ\left(I \otimes b^{-+}\right)=I,
\end{aligned}
$$

the value of a planar graph as a tensor is invariant under isotopy of the graph.

In the combinatorial $A_{2}$ spider, a (monomial) web is defined as any composition of tensor products of $b$ 's, $t$ 's, and $\sigma$ 's. Any such web is denoted by an oriented graph in a disk with trivalent and univalent vertices, and possibly closed loops, such that the edges are either all out or all in at the trivalent vertices, and such that the univalent vertices are at the boundary of the disk.

By the fundamental theorem of invariant theory, the set of all monomial webs with any given boundary spans the corresponding set of invariants. 
Moreover, the following relations hold:

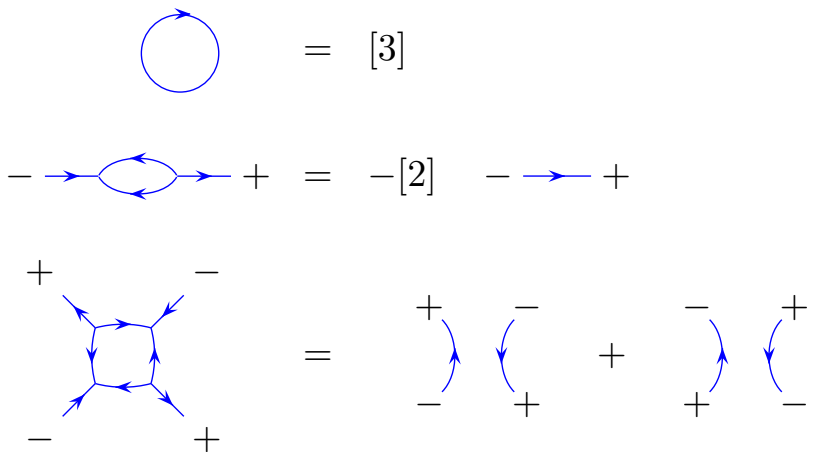

Thus, the set of non-elliptic webs, i.e., webs such that all internal faces have at least six sides, also spans. It is a fundamental result that the set of non-elliptic webs is a basis of each invariant space [5]. These are the web bases that we will compare to the dual canonical bases.

\section{State sums.}

In the given bases of $V^{+}$and $V^{-}$, the $b, t$, and $\sigma$ tensors have matrices, and any monomial web can be evaluated by the usual linear algebra method of summing over indices of these matrices. Such an expansion is equivalent to a state sum in the sense of statistical mechanics. Given a monomial web $w$, we first divide each edge into segments whose edges are the points where the edge has a horizontal tangent. A state is then a function from the segments to the set $\{-1,0,1\}$. The weight of a state at each trivalent vertex or horizontal tangent is a matrix entry of the corresponding $b, t$, or $\sigma$ tensor. A boundary state is a function from just those segments with univalent vertices to $\{-1,0,1\}$. The weight of a boundary state is then defined as the total weight of all extensions of the boundary state to a state of the entire graph. The weights of the boundary state are then the coefficients of $w$ expanded in the tensor product basis.

We will abbreviate a state in a state sum by flow lines. A collection of flow lines in a monomial web $w$ is a subgraph that contains exactly two of the three edges incident to each trivalent vertex. Each flow line is oriented; this orientation need not agree with the orientation of $w$. Every segment of an edge disjoint from a flow line has the state 0 . If a flow line is oriented downward along a segment, the segment has the state 1 , while if it is oriented upward, the segment has the state -1 . In this way, flow lines represent precisely those states with non-zero weight. 
A second convenience for computing state sums is to introduce the linear endomorphisms

$$
\begin{aligned}
& t_{-}^{++}: V^{-} \rightarrow V^{+} \otimes V^{+} \\
& t_{+}^{--}: V^{+} \rightarrow V^{-} \otimes V^{-} \\
& t_{--}^{+}: V^{-} \otimes V^{-} \rightarrow V^{+} \\
& t_{++}^{-}: V^{+} \otimes V^{+} \rightarrow V^{-} .
\end{aligned}
$$

They may be defined as the webs:
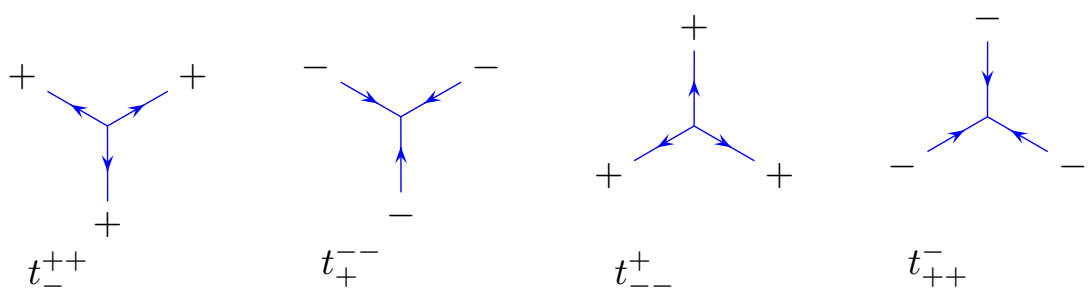

(Note that here, as before, the signs of the tensor subscripts are opposite to the signs at the bottom of the webs, because $\operatorname{Hom}\left(V, V^{\prime}\right) \cong \operatorname{Inv}\left(V^{*} \otimes V^{\prime}\right)$.) Their coefficients are given by:

$$
\begin{aligned}
t_{-}^{++} e_{1}^{-} & =e_{1}^{+} \otimes e_{0}^{+}+v^{-1} e_{0}^{+} \otimes e_{1}^{+} \\
t_{-}^{++} e_{0}^{-} & =e_{1}^{+} \otimes e_{-1}^{+}+v^{-1} e_{-1}^{+} \otimes e_{1}^{+} \\
t_{-}^{++} e_{-1}^{-} & =e_{0}^{+} \otimes e_{-1}^{+}+v^{-1} e_{-1}^{+} \otimes e_{0}^{+}
\end{aligned}
$$

and

$$
\begin{aligned}
t_{--}^{+}\left(e_{1}^{-} \otimes e_{0}^{-}\right) & =v e_{1}^{+} & t_{--}^{+}\left(e_{0}^{-} \otimes e_{1}^{-}\right) & =e_{1}^{+} \\
t_{--}^{+}\left(e_{1}^{-} \otimes e_{-1}^{-}\right) & =v e_{0}^{+} & t_{--}^{+}\left(e_{-1}^{-} \otimes e_{1}^{-}\right) & =e_{0}^{+} \\
t_{--}^{+}\left(e_{0}^{-} \otimes e_{-1}^{-}\right) & =v e_{-1}^{+} & t_{--}^{+}\left(e_{-1}^{-} \otimes e_{0}^{-}\right) & =e_{-1}^{+} .
\end{aligned}
$$

As before, all combinations not listed are 0 . The formulas for $t_{++}^{-}$and $t_{+}^{--}$ are the same; one just switches +'s and -'s. These endomorphisms have graphs that are Y's and $\lambda$ 's; their weights may be abbreviated with flow lines according to the following chart:

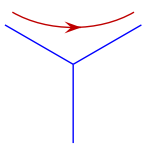

1

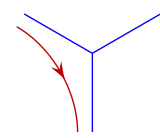

1

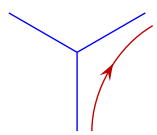

1

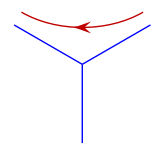

$v^{-1}$
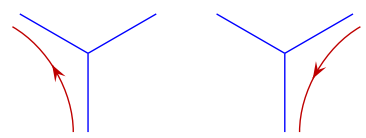

$v^{-1}$ 


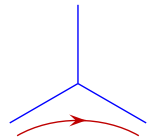

1

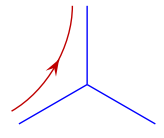

1

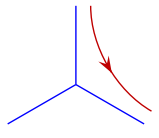

1

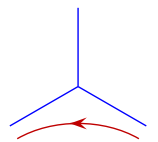

$v$

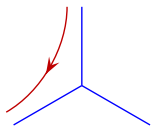

$v$

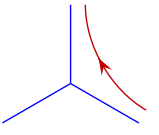

$v$

Figure (3).

For completeness, we give also give a chart of weights of the $b$ 's and $\sigma$ 's:

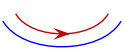

1

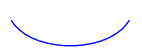

$v^{-1}$

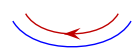

$v^{-2}$

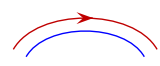

1

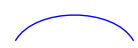

$v$

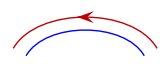

$v^{2}$

Figure (4).

As an example of computing a state sum using flow lines, the following are the only two non-zero states with boundary $0,0,0,0,0,1,-1$ in a certain web $w$ :
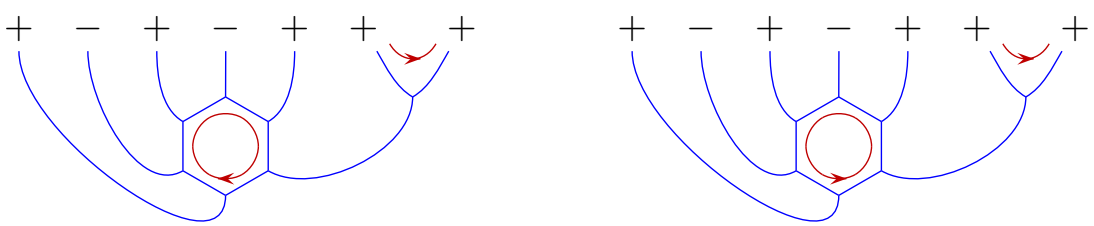

Since the weights of these states are $v^{-1}$ and $v^{-3}$, we therefore conclude that the coefficient of

$$
e_{0}^{+} \otimes e_{0}^{-} \otimes e_{0}^{+} \otimes e_{0}^{-} \otimes e_{0}^{+} \otimes e_{1}^{+} \otimes e_{-1}^{+}
$$

in $w$ is $v^{-1}+v^{-3}$ in the tensor product basis.

Finally, note that the weight of any state of any monomial web is either a power of $v$ or zero. Thus, weights cannot cancel in state sums, and any state sum takes values in $\mathbb{N}\left[v, v^{-1}\right]$.

\section{From paths and strings to non-elliptic webs.}

In order to compare the web and dual canonical bases, we must enumerate non-elliptic webs by certain strings of elements of $\{-1,0,1\}$, namely those that correspond to weight lattice paths confined to a Weyl chamber of $\operatorname{sl}(3)$.

More precisely, let $S=s_{1}, \ldots, s_{n}$ be a string of signs, and let $J=$ $j_{1}, \ldots, j_{n}$ be a string of states chosen from $\{-1,0,1\}$. Each vector $e_{j_{k}}^{s_{k}}$ has a weight $\mu_{k}$, and we may define a path $0=\pi_{0}, \pi_{1}, \pi_{2}, \ldots, \pi_{n}$ in the weight lattice of $\operatorname{sl}(3)$ such that $\pi_{k}=\mu_{k}+\pi_{k-1}$. The dominant Weyl chamber 
is defined as the subset of the weight lattice consisting of positive integral linear combinations of the weights $\mu^{+}$and $\mu^{-}$of $e_{1}^{+}$and $e_{1}^{-}$. It is well-known that, for fixed $s_{1}, \ldots, s_{n}$, the number of strings $j_{1}, \ldots, j_{n}$ that produce a lattice path in the dominant Weyl chamber from the origin to itself equals

$$
\operatorname{dim} \operatorname{Inv}\left(V^{s_{1}} \otimes V^{s_{2}} \otimes \ldots \otimes V^{s_{n}}\right) .
$$

Call such lattice paths dominant.

Given a sign string and a string of states, we define a web by inductive rules called the growth algorithm:

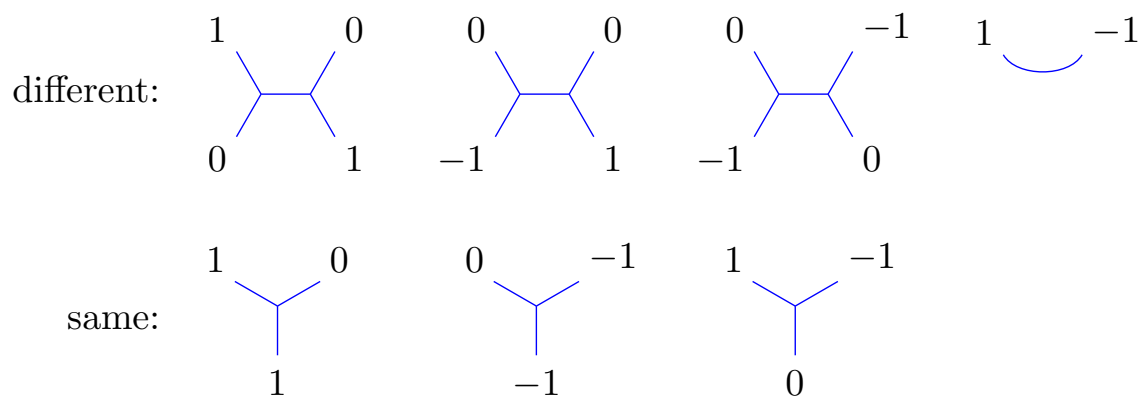

Initially, the web consists of parallel strands whose orientations are given by the sign string. The rules indicate that if the state string admits a substring of the type appearing at the top in one of the cases (taken from the top row if the two signs are different and from the bottom row if they are the same), we should concatenate the corresponding web and replace the substrings with what appears at the bottom of the web. If none of the patterns at the top appear anywhere, the growth algorithm stops. For example, the growth algorithm converts the sign string +-+-+++ and the state string $1,1,0,0,-1,0,-1$ to the web:

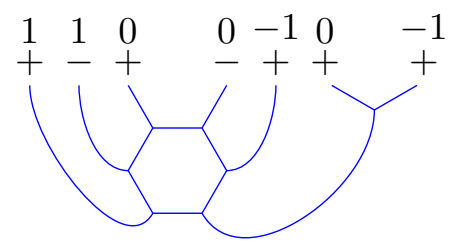

In this case the growth algorithm continues until the sign and state strings have length 0 .

The validity of the growth algorithm rests on the following lemmas.

Lemma 1. Given any sign and state string, the web produced by the growth algorithm does not depend on the order in which the substrings are replaced.

Proof. (Sketch) The proof is by induction. Order state strings by their length; if two state strings have the same length, order them lexicographically. A minimal counterexample consisting of a sign string $S$ and a state 
string $J$ must have two different replaceable substrings that ultimately result in two different webs; given two such replacements $r_{1}$ and $r_{2}$, let $w_{1}$ and $w_{2}$ be the two webs that result, and let $S_{1}$ and $S_{2}$ and $J_{1}$ and $J_{2}$ be the sign and state strings that result. The pairs $\left(S_{1}, J_{1}\right)$ and $\left(S_{2}, J_{2}\right)$ are not counterexamples because $J_{1}$ and $J_{2}$ come before $J$; therefore the growth algorithm is order-independent for both of these strings. To obtain a contradiction, it suffices to complete a diamond by finding a pair $\left(S_{3}, J_{3}\right)$ which can be obtained from either $\left(S_{1}, J_{1}\right)$ or $\left(S_{2}, J_{2}\right)$ by the growth algorithm, for example:

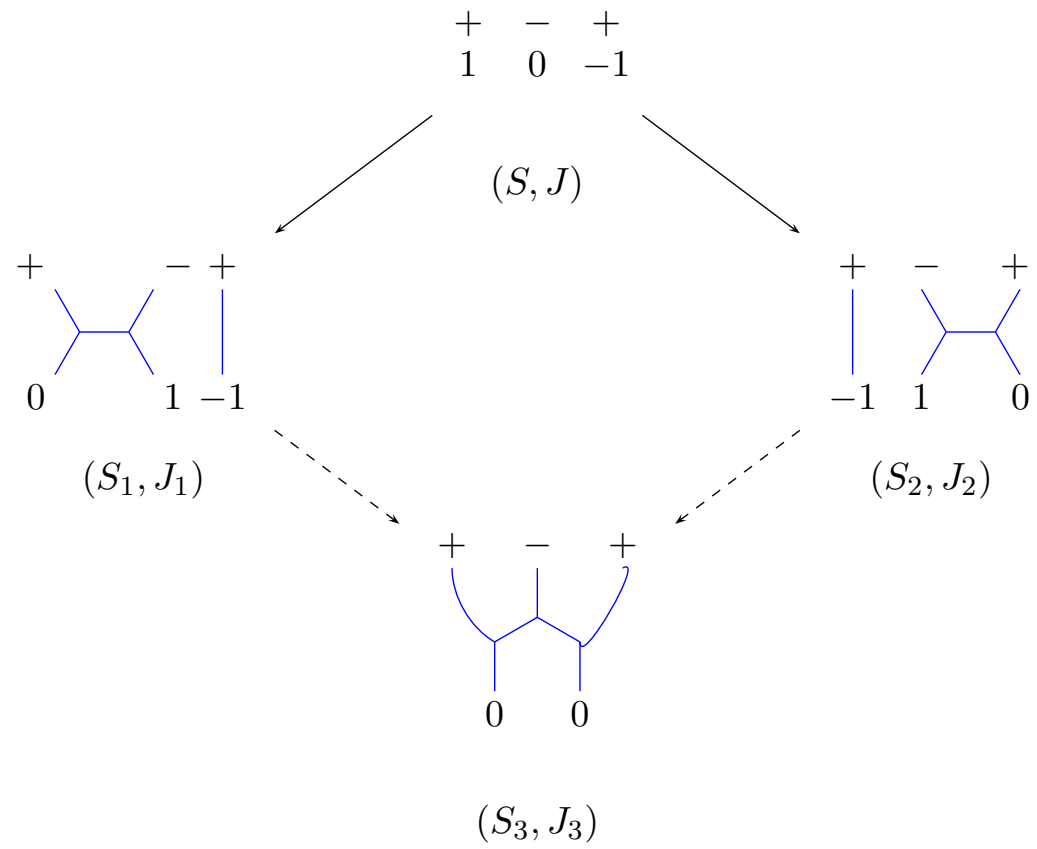

Figure (5).

If the replacements $r_{1}$ and $r_{2}$ have disjoint substrings, then we can trivially complete the diamond by appling $r_{2}$ after $r_{1}$ and vice-versa. There is a short list of cases in which they are not disjoint, and we can complete the diamond on a case-by-case basis. Figure (5) gives one of the cases.

Lemma 2. Any web produced by the growth algorithm is non-elliptic.

Proof. (Sketch) In the growth rules, an internal face can only be "born" with a rule that produces an $\mathrm{H}$. The only way to obtain a 2 -sided face would be 
to close off the face immediately with a U. However, the indices that result from attaching an $\mathrm{H}$ rule out this possibility.

A square face can be ruled out by a more complicated version of the same reasoning. A square might hypothetically have one of four possible histories:
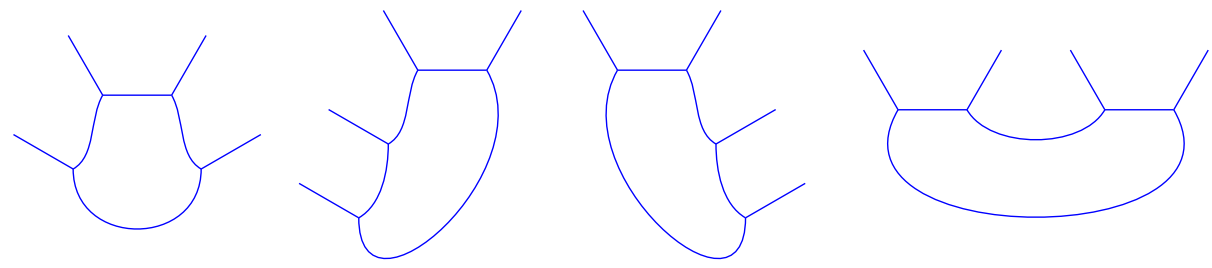

In the first three cases, the extra vertices may belong either to H's or Y's produced by the growth algorithm at adjacent locations. Working backwards from the final U, one quickly concludes that none of the histories are possible.

Lemma 3. If a sign and state string correspond to a dominant lattice path, then the growth algorithm does not terminate until the strings have length 0.

Proof. (Sketch) The proof is again by induction on length and lexicographic order. Observe that the growth algorithm only terminates at a non-decreasing state string (and only then when all positions $k$ such that $j_{k}=0$ have the same sign $s_{k}$ ). On the other hand, the state string of a dominant path of length greater than 0 must begin with 1 and end with -1 . Thus, it suffices to show that a growth rule applied to a dominant path produces another dominant path. None of the 14 growth rules change the endpoints of the corresponding lattice path, and each of them either reduces the set of vertices it visits or modifies it in a way that cannot lead to an excursion outside of the dominant Weyl chamber. For example, a growth rule that produces a Y replaces two consecutive steps of the path by one step:
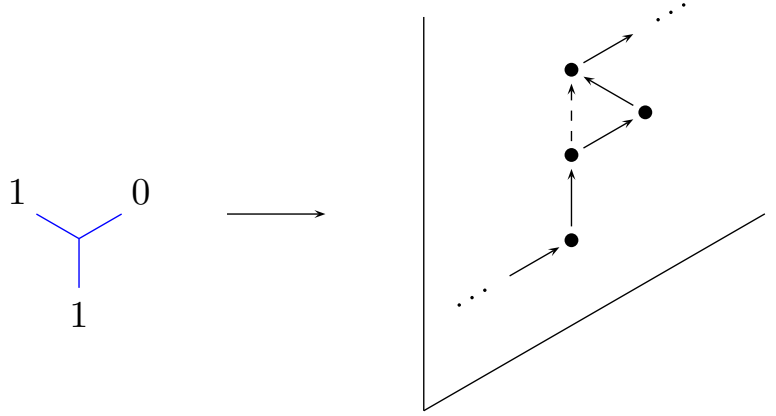

The converse of Lemma 3 also holds [4].

The growth algorithm has a notable inverse for dominant paths. Let $w$ be a non-elliptic web. Given points $P$ and $Q$ on the boundary of $w$ lying 
between endpoints, a minimal cut path is a transversely oriented arc from $P$ to $Q$ which is transverse to $w$ and which crosses as few strands as possible:

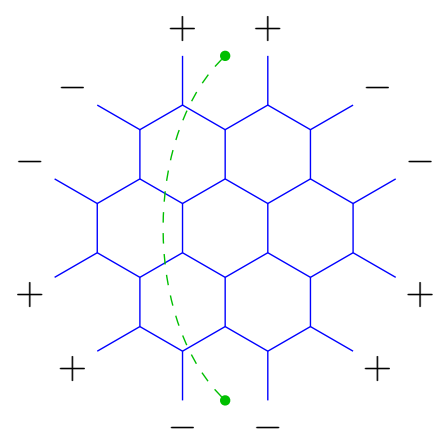

The weight of a minimal cut path is $a \mu^{+}+b \mu^{-}$if the cut path crosses $a$ strands whose orientations agree with that of the arc and $b$ strands whose orientations disagree. Although minimal cut paths are not necessarily unique, their weights are [5]. Moreover, any two minimal cut paths are connected by a sequence of H-moves:

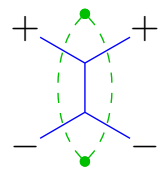

Now let $w$ be a non-elliptic web with $n$ endpoints that are linearly ordered (rather than cyclically ordered) and lie above $w$, as might be produced by the growth algorithm. Let $P$ be a point below $w$, and let $Q_{0}, Q_{1}, \ldots, Q_{n}$ be points that alternate with the endpoints of $w$. Let $\pi_{k}$ be the weight of a minimal cut path from $P$ to $Q_{k}$ :

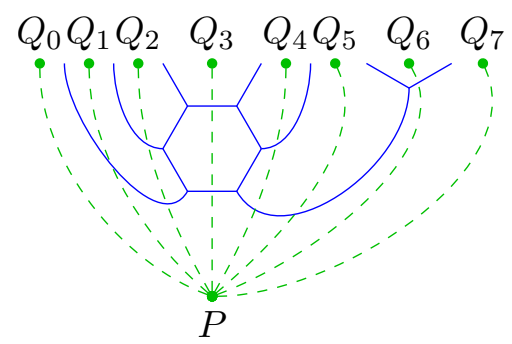

The sequence $0=\pi_{0}, \pi_{1}, \ldots, \pi_{n}=0$ is a dominant lattice path [5]. Each successive difference $\pi_{k}-\pi_{k-1}$ is the weight of some $e_{j_{k}}^{s_{k}}$, so we can reconstruct both a sign string (which is given directly by the boundary of $w$ ) and a state string from the web $w$. Call this procedure the minimal cut path algorithm.

Proposition 1. The minimal cut path and growth algorithms are inverses. 
Proof. For each fixed sign string $S$, let $w_{J}^{S}$ be the web produced by the growth algorithm from the state string $J=j_{1}, \ldots, j_{n}$, and let $m(w)$ be the state string produced by the minimal cut path algorithm from the basis web $w$. We will show that $m \circ g=I$. Since $m$ is a bijection [5], it follows that $g \circ m=I$ also.

We extend the growth rules to create a system of flow lines and minimal cut paths along with the basis web. The extension is given by the following diagrams:

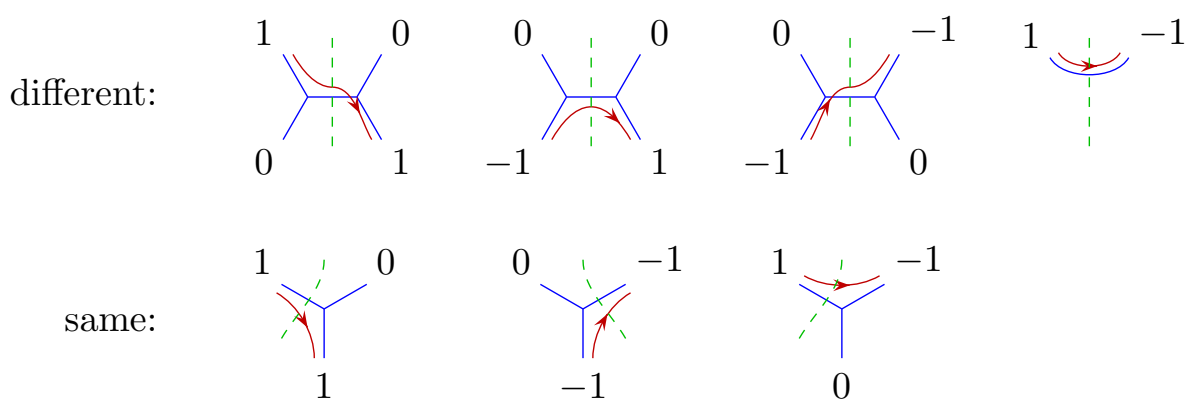

Figure (6).

In each case, cut paths may merge, so that at any step after the first one, a single cut path represented in the diagram may be replaced by many parallel cut paths. In the final result, the cut paths are all minimal by the principle that MIN CUT $\geq$ MAX FLOW. I.e., if the sum of the indices to the left of a given cut path $\gamma$ is $m$, then by conservation of flow, $\gamma$ must cross at least $m$ flow lines. By construction, any strand that $\gamma$ cross has flow on it and all flow across $\gamma$ is to the right; therefore $\gamma$ must cross exactly $m$ flow lines.

Since the chosen cut paths are minimal, it is routine to show by induction on the number of steps in the growth algorithm that the minimal cut path algorithm reconstructs the original state string.

Remark. A well-known theorem in the theory of linear programming is often summarized by the maxim MIN CUT = MAX FLOW. However, if we applied this theorem directly, we would have to allow the possibility of fractional flow lines. Thus, we have shown in our case that the maximal flow in the linear sense can be achieved combinatorially without using flow lines with forks.

Reference [5] demonstrated that the set of non-elliptic webs is a $\mathbb{C}(q)$ basis of $\operatorname{Inv}\left(V^{s_{1}} \otimes \ldots \otimes V^{s_{n}}\right)$. Proposition 1 gives a way to index this basis by dominant paths. Here we can obtain a stronger result. If $S=s_{1}, \ldots, s_{n}$ is a sign string and $J=j_{1}, \ldots, j_{n}$ is a state string, define $e_{J}^{S}$ by:

$$
e_{J}^{S}=e_{j_{1}}^{s_{1}} \otimes e_{j_{2}}^{s_{2}} \otimes \ldots \otimes e_{j_{n}}^{s_{n}} .
$$


Theorem 2. The tensor $w_{J}^{S}$ expands as

$$
w_{J}^{S}=e_{J}^{S}+\sum_{J^{\prime}<J} c\left(S, J, J^{\prime}\right) e_{J^{\prime}}^{S}
$$

for some coefficients $c\left(S, J, J^{\prime}\right) \in \mathbb{N}\left[v, v^{-1}\right]$, where the state strings $J$ and $J^{\prime}$ are ordered lexicographically.

It follows that the non-elliptic webs are a $\mathbb{Z}\left[v, v^{-1}\right]$-basis of the invariant spaces in which they live.

Proof. The result follows from the existence of the minimal cut paths. Recall that the coefficients $c\left(S, J, J^{\prime}\right)$ are state sums. For each $k$, the cut path $\gamma$ from $P$ to $Q_{k}$ cuts $m$ strands, where $m=\sum_{\ell=1}^{k} j_{\ell}$. But if $J^{\prime}>J$, we can choose the first $k$ such that $j_{k} \neq j_{k}^{\prime}$; in this case, $j_{k}^{\prime}>j_{k}$. The cut path $\gamma$ must cut at least $\sum_{\ell=1}^{k} j_{\ell}^{\prime}>m$ flow lines in any state contributing to $c\left(S, J, J^{\prime}\right)$. But this is impossible, since $\gamma$ only cuts $m$ strands.

The case $J^{\prime}=J$ is more delicate. We claim that only one non-zero state contributes to $c(S, J, J)$, namely the state $x$ constructed in Proposition 1 . Since MIN CUT = MAX FLOW by this proposition, every edge which intersects any minimal cut path must carry flow in every non-zero state. The claim follows if we can show that minimal cut paths meet every edge that carries flow in the state $x$.

Consider two cut paths from $P$ to adjacent endpoints $Q_{k}$ and $Q_{k+1}$. If we move the cut paths as close together as possible using H-moves, then by a curvature argument [5], there are only three possibilities for the portion of the web between them:
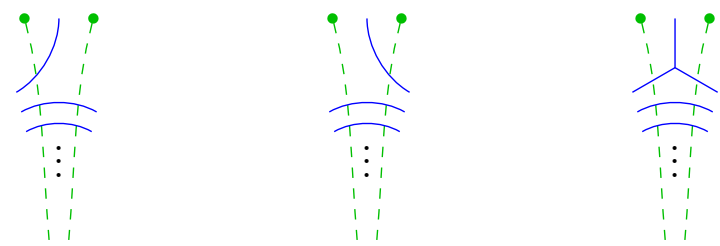

In each case, there are no edges with flow between the two cut paths. At the same time, when one performs an H-move on a cut path, the path hops over a single edge which does not carry flow. Thus, the set of all cut paths intersects every edge with flow.

It remains to show that the weight of the state $x$ is 1 . This follows from comparing the extended growth rules in Figure (6) to the weights in Figures (3) and (4).

Given a sign string $S$ and a non-dominant state string $J$, the growth rules produce a new sign string $S^{\prime}$, a state string $J^{\prime}$, and a web $w_{J}^{S} \in$ 
$\operatorname{Hom}\left(V^{S^{\prime}}, V^{S}\right)$. Theorem 2 generalizes to the vectors $w_{J}^{S}\left(e_{J^{\prime}}^{S^{\prime}}\right)$ to produce a web basis for all of $V^{S}$ [4].

\section{The dual canonical axioms.}

In this section, we will give axioms that uniquely determine the dual canonical bases of invariant spaces. The first axiom involves a certain operator $\bar{\Theta} \in U_{q}(\operatorname{sl}(3)) \hat{\otimes} U_{q}(\operatorname{sl}(3))$, where " $\hat{\otimes}$ " is a certain topological tensor product $[\mathbf{1}, \mathbf{6}]$. For each tensor product $V=V^{S}$, we define a $v$-antilinear endomorphism $\Phi=\Phi^{S}$ inductively by the rule

$$
\Phi^{S S^{\prime}}\left(e^{S} \otimes e^{S^{\prime}}\right)=\bar{\Theta}\left(\Phi^{S}\left(e^{S}\right) \otimes \Phi^{S^{\prime}}\left(e^{S^{\prime}}\right)\right),
$$

where $e^{S} \in V^{S}$ and $e^{S^{\prime}} \in V^{S^{\prime}}$. (By $v$-antilinearity, we mean that $\Phi$ is $\mathbb{C}$ linear and that $\Phi(v e)=v^{-1} \Phi(e)$.) The action of $\Phi$ on $V^{+}$and $V^{-}$is defined by the stipulation that it fixes $\left\{v_{i}^{ \pm}\right\}$. Remarkably, the properties of $\bar{\Theta}$ imply that this definition is consistent.

Theorem 3 (Lusztig). For any sign string $S=s_{1}, \ldots, s_{n}$ and any state string $J=j_{1}, \ldots, j_{n}$, there is a unique element

$$
e_{\circlearrowleft J}^{S}=e_{j_{1}}^{s_{1}} \nabla e_{j_{2}}^{s_{2}} \oslash \ldots \varnothing e_{j_{n}}^{s_{n}} \in V^{S}
$$

which is invariant under $\Phi$ and such that

$$
e_{j_{1}}^{s_{1}} \nabla e_{j_{2}}^{s_{2}} \varnothing \ldots \varnothing e_{j_{n}}^{s_{n}}=e_{J}^{S}+\sum_{J^{\prime}} c\left(S, J, J^{\prime}\right) e_{J^{\prime}}^{S}
$$

with $c\left(S, J, J^{\prime}\right) \in v^{-1} \mathbb{Z}\left[v^{-1}\right]$ (the negative-exponent property).

Clearly, $\left\{e_{\circlearrowleft J}^{S}\right\}$ is a basis of $V^{S}$, the dual canonical basis. It is less clear, but nevertheless true, that the subset of $\left\{e_{\odot J}^{S}\right\}$ indexed by dominant paths is a basis of $\operatorname{Inv}\left(V^{S}\right)$ [6, Sec. 27.2.5].

It remains to determine when $w_{J}^{S}=e_{\bigcirc J}^{S}$, i.e., when basis webs are dual canonical.

\section{Early agreement.}

By inspection, the empty web $w_{\emptyset}^{\emptyset}=1$ and the webs $w_{1,-1}^{-+}=b^{-+}$and $w_{1,-1}^{+-}=$ $b^{+-}$have the negative-exponent property. They are therefore dual canonical, because there must be a dual canonical vector in the one-dimensional space of invariants in which they lie, and they do not retain the negative-exponent property after rescaling. The same argument applies to $t^{+++}$and $t^{---}$.

Proposition 2. Every basis web $w_{J}^{S}$ is invariant under $\Phi$.

Proof. We will actually prove that every morphism made out of $b$ 's, t's, and $\sigma$ 's, in other words every (monomial) web interpreted arbitrarily as an element of $\operatorname{Hom}\left(V^{S}, V^{S^{\prime}}\right)$, is equivariant under $\Phi$. For this purpose, it is 
convenient to define $\Phi=\Phi^{\emptyset}$ for a 0 -fold tensor product as $v$-conjugation; $\Phi(v)=v^{-1}$.

Clearly, the identity $I$ is equivariant.

Since the $b$ 's and the $t$ 's are dual canonical, they are invariant under $\Phi$, or equivariant as morphisms. Let us assume for a moment that the $\sigma$ 's are equivariant also. If $L$ and $L^{\prime}$ are both equivariant under both $\Phi$, then so is their composition if they can be composed. If they are also both equivariant under the action of $U_{q}(\operatorname{sl}(3))$, as any web is, then $L \otimes L^{\prime}$ intertwines $\Phi \otimes \Phi$, and it also intertwines $\bar{\Theta} \in U_{q}(\operatorname{sl}(3)) \hat{\otimes} U_{q}(\operatorname{sl}(3))$. It therefore intertwines $\Phi$. The proposition follows by induction, decomposing an arbitrary web as a tensor product or composition of simpler pieces.

The equivariance of $\sigma$ 's follows from equations (1) and a reversal of the previous argument. The map $\Phi$ intertwines the identity; pushing $\Phi$ from right to left on the left side of the first equation, we conclude that

$$
\left(I \otimes\left(\sigma_{-+} \circ \Phi^{-+}\right)\right) \circ\left(b^{+-} \otimes I\right)=\left(I \otimes\left(\Phi^{\emptyset} \circ \sigma_{-+}\right)\right) \circ\left(b^{+-} \otimes I\right) .
$$

Because $b^{+-}$is non-singular, this implies

$$
\sigma_{-+} \circ \Phi^{-+}=\Phi^{\emptyset} \circ \sigma_{-+}
$$

The same argument applies to $\sigma_{+-}$.

For a general basis web $w_{J}^{S}$, each state has some weight $v^{n}$; call $n$ the exponent of the state. The web $w_{J}^{S}$ has a distinguished state with weight 1 , namely the unique state with boundary $J$. By Theorem 2, we can call this state the leading state. It is dual canonical if and only if every non-leading state has negative exponent. In the following discussion, let $w$ be a basis web which is not dual canonical and which has as few endpoints as possible.

Proposition 3. A minimal counterexample $w$ is connected and does not have a $Y$ or a double $H$ at the boundary:
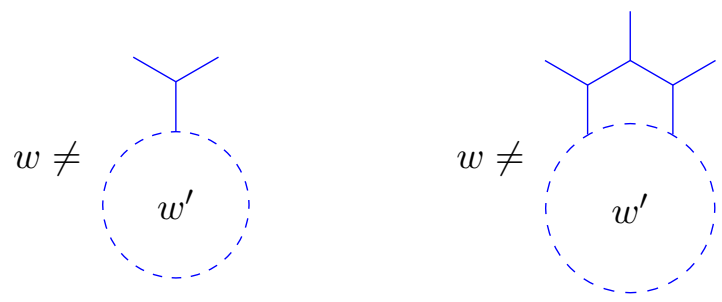

Proof. Suppose, to the contrary, that $w$ is the disjoint union of $w^{\prime}$ and $w^{\prime \prime}$. A state of $w$ restricts to a state of $w^{\prime}$ and a state of $w^{\prime \prime}$, and the weights multiply. Since only one state of either $w^{\prime}$ or $w^{\prime \prime}$ has weight $v^{n}$ with $n \geq 0$, the same is true of $w$. Thus, $w$ has the negative-exponent property.

Suppose that $w$ has a $\mathrm{Y}$ at the boundary. Let $w^{\prime}$ be $w$ without the $\mathrm{Y}$. By inspection of $t_{-}^{++}$and $t_{+}^{--}$, the $\mathrm{Y}$ has six possible states, three with weight 1 and three with weight $v^{-1}$. At the same time, any state of $w^{\prime}$ has negative 
exponent except for the leading state, which has exponent 0 . Thus, the only way that $w$ can fail to have the negative-exponent property is if there are two distinct states which have weight 1 on both $w^{\prime}$ and the Y. But since the three states of the $\mathrm{Y}$ have different states on the edge that it shares with $w^{\prime}$, this is not possible.

Suppose finally that $w$ has a double $\mathrm{H}$. The argument here is essentially the same as for a $\mathrm{Y}$, but more complicated. We arrange the double $\mathrm{H}$ as a composition of one $\lambda$ and two Y's, as above. Then one can check on a caseby-case basis that there it has no states with positive exponent and twelve states with weight 1 . These twelve states run through all nine possibilities for the states of the two bottom edges, with three repeats. The three repeats are 1 and $0 ; 1$ and -1 ; and 0 and -1 . Again let $w^{\prime}$ be the remainder. If $w$ had two states with weight 1 , then because $w^{\prime}$ has the negative-exponent property, the two states can only differ in the double H. Moreover, the states at the bottom extend to the leading state of $w^{\prime}$, so $w^{\prime}$ can be constructed using the growth rules. In particular, we can apply a growth rule to the two edges of the double $\mathrm{H}$ to conclude that $w^{\prime}$ has a $\mathrm{Y}$ here. Together, they make a square:

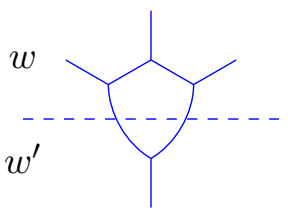

The square is an elliptic face of $w$, a contradiction.

Proposition 3 establishes the positive part of Theorem 1, since by a curvature argument, a connected basis web with at least one vertex and with no Y's at the boundary must have at least six H's, and no two of the H's can share vertices. Moreover, one can eliminate all but one web with twelve vertices, a web which as it happens is the first counterexample.

\section{Counterexamples.}

It is easier to demonstrate that counterexamples eventually appear than to find or verify any particularly small counterexamples. Consider a hexagon 
made of three $\lambda$ 's and three Y's that is part of some larger web:

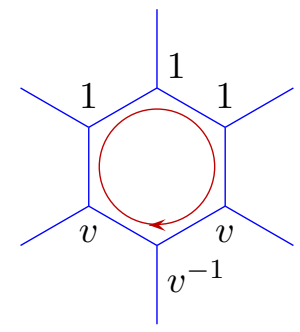

If the hexagon has a closed, clockwise flow line, as indicated, then its overall weight is $v$.

A large flat web locally resembles the tiling of the plane by regular hexagons. The number of such hexagons can grow quadratically in the length of the periphery, and we can put flow loops on one third of the hexagons to form a valid state. In the limit, the exponent of any such state must be positive. Thus, not all basis webs have the negative exponent property.

The smallest counterexample is similar to the asymptotic ones:

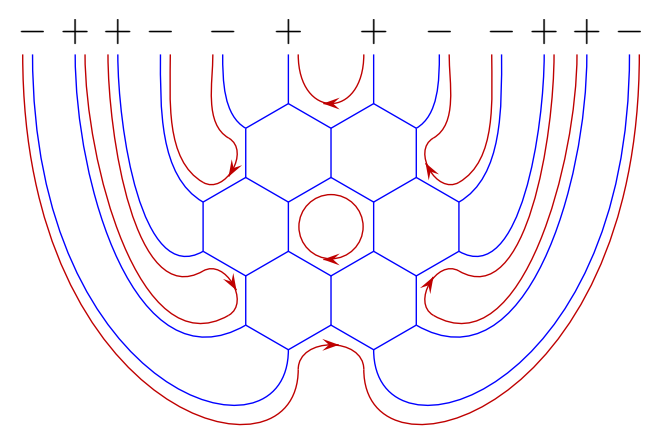

Figure (7).

One can compute the weight of this state by noting that, besides the complete hexagon in the middle, the flow lines make three more hexagons divided into halves, and in addition there are two right-ward pointing U-turns. Thus the weight is $v^{4} v^{-4}=1$. If one rotates the web, another state with weight 
1 appears:

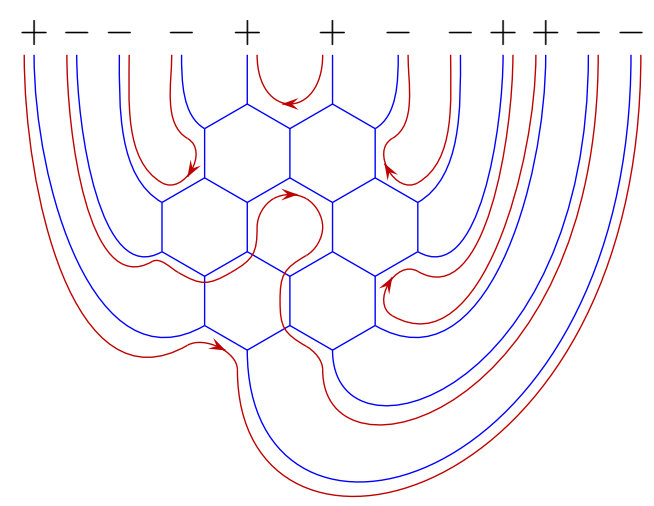

The fact that neither web is dual canonical is consistent with the fact that both web bases and dual canonical bases are preserved by cyclic permutation of tensor factors.

Theorem 4. The web
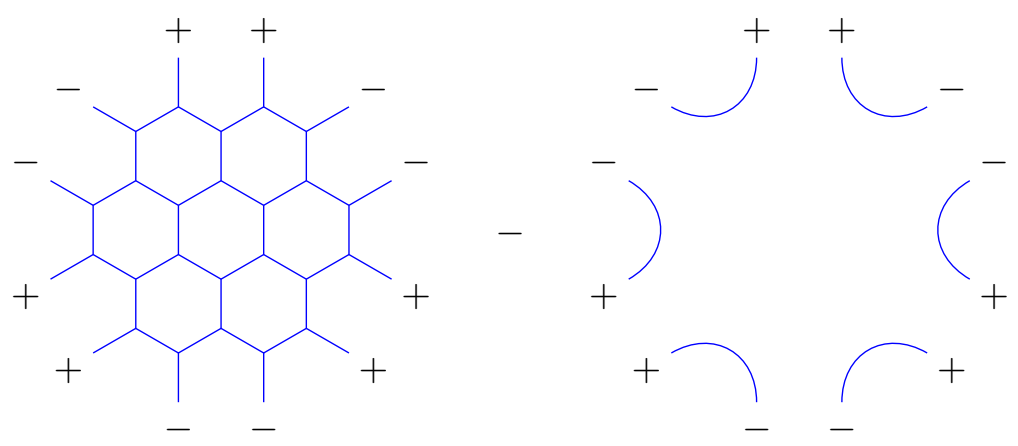

is dual canonical.

Proof. (Sketch) Removing an $\mathrm{H}$ from the counterexample $w$ in Figure (7) produces a non-counterexample $w^{\prime}$ by Proposition 3. A hypothetical state $x$ with non-negative exponent must either have weight $v$ or 1 on the $\mathrm{H}$; in the latter case, the state $x$ must restrict to the leading state of $w^{\prime}$ and yet differ from the leading state of $w$. A combinatorial investigation reveals that the second alternative is impossible. The only possibility for the local state for each of the six H's forces $x$ to be the state given in Figure (7). Thus we can subtract off another basis web, which happens to be dual canonical, to eliminate this term and recover the negative-exponent property.

\section{What is to be done?}

What can one conclude from the fact that the web bases are not dual canonical? First, we argue that the web bases are nevertheless interesting. They are useful for computing quantum link invariants, and they may be useful for 
computing $6 j$-symbols along the lines of Masbaum and Vogel's computation for $\operatorname{sl}(2)[7]$.

In light of Theorem 4, it is possible that web bases can somehow be understood using perverse sheaves that are the same as those related to canonical bases except for certain salient subsheaves or stalks.

\section{Higher-rank spiders.}

The main open problem concerning spiders and web bases is to generalize the combinatorial rank two spiders to higher rank. If the web bases were dual canonical, it would have given an immediate definition, albeit a very different one from the one given in Reference [5]. Since it is not the case, we outline a possible alternative approach to such a generalization.

Calculations in rank 2 spiders exhibit many elements of the CoxeterWeyl geometry of the corresponding affine Weyl group, this phenomenon depends on the coincidence that the dimension of a web equals the rank of the Lie algebra. Nevertheless, it is implausible that a higher-rank spider would involve higher-dimensional webs, because quantum groups, irrespective of their rank, are fundamentally related to low-dimensional geometry and topology. Moreover, the $A_{1}$ or Temperley-Lieb spider has 2-dimensional webs even though the Lie algebra has rank 1.

Informally, a large flat web in any of the three rank 2 spiders resembles the Voronoi tiling of the plane associated to the weight lattice. More specifically, if one generically immerses a disk in the plane of a weight lattice, then the pull-back of the edges and vertices of the Voronoi tiling forms a valid nonelliptic web:
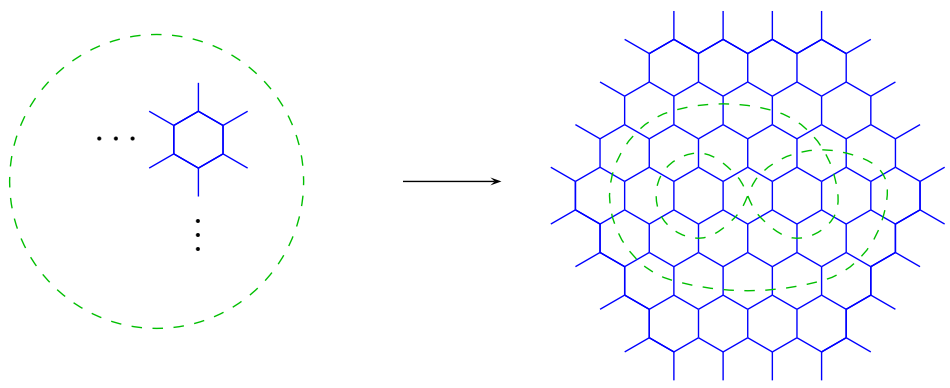

Similarly, in the rank 1 case, there is a weight-lattice Voronoi tiling of the line by line segments. If one submerses a disk, the inverse image of the endpoints of these line segments is some 1-manifold, which is then a basis web in the $A_{1}$ spider.

Thus, we may hypothesize that a web in a rank $n$ spider resembles the inverse image of a weight-lattice Voronoi tiling under an immersion of a disk in $\mathbb{R}^{n}$. An essential ingredient, which is present in the rank 1 and 2 cases, is that the codimension 1 faces of such a Voronoi tiling are labelled by 
fundamental irreducible representations. More concretely, if $\mu_{1}$ and $\mu_{2}$ are two Voronoi adjacent points in the weight lattice, there is a unique dominant weight $\lambda$ which is conjugate to $\mu_{1}-\mu_{2}$. The codimension 1 face separating $\mu_{1}$ from $\mu_{2}$ pulls back under an immersion of a disk to an edge; this edge might then be labelled by the representation $V(\lambda)$.

For example, the weight lattice of the Lie algebra $A_{3}=\operatorname{sl}(4)$ is the BCC lattice. The Voronoi region of a lattice point is a 14-side snub octahedron; following the convention just described, the six-sided faces are labelled with the defining representation $V$ of $\operatorname{sl}(4)$ and its dual $V^{*}$, while the four-side faces are labelled by the six-dimensional representation $\bigwedge^{2} V$. The incidence of the faces suggests vertices of the form:
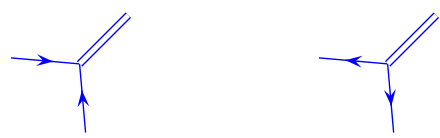

Here an oriented edge is one labelled by $V$ or $V^{*}$, while a double edge is one labelled by $\bigwedge^{2} V$.

Both of these hypothetical vertices correspond to invariant tensors which are unique up to a scalar factor. One can then consider relations which these tensors satisfy. These include some elliptic-looking relations such as:

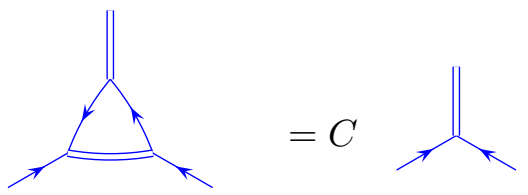

But there is also the relation:

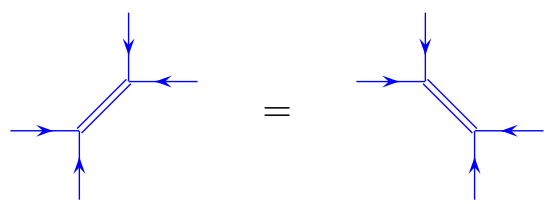

Figure (8). 
This relation (and its dual) can perhaps be motivated by a homotopy of a disk across a vertex of the Voronoi tiling. And there are the relations:
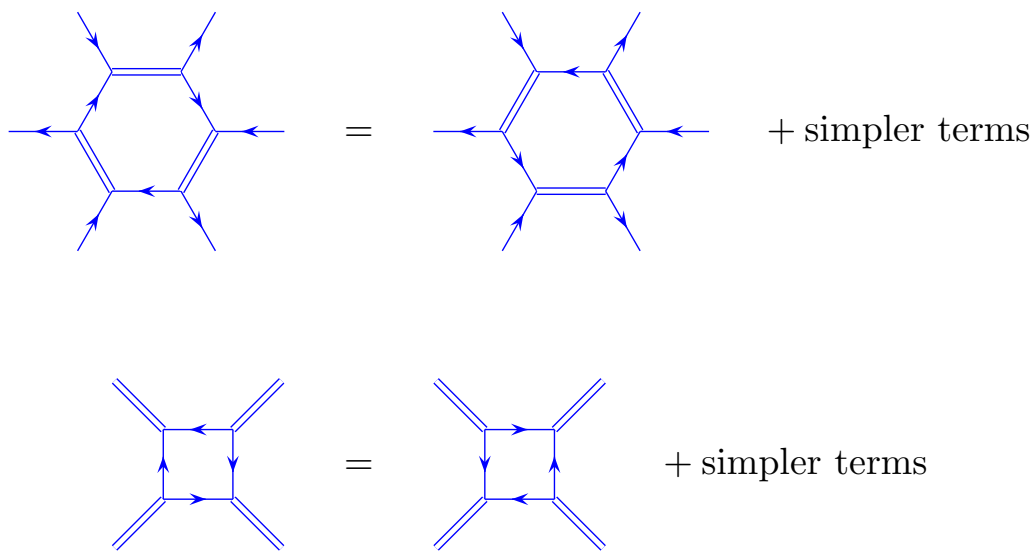

These relations appear to be related to the faces of the snub octahedron.

Despite these suggestive relations, we do not know how to put them into a coherent whole. Some relations that one might predict from Voronoi geometry do not hold. For example, if one maintains that the structure of a vertex in the Voronoi tiling predicts relation (8), then presumably it would also predict a relation between the two webs:
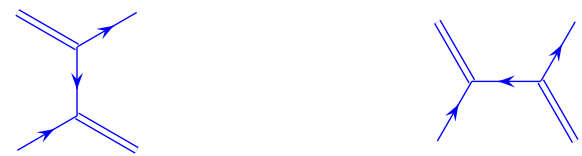

However, these two webs are linearly independent.

Ideally, we would like an explicit presentation for the representation category of a Lie algebra which is akin to the Serre relations for the Lie algebra itself.

\section{References}

[1] I. Frenkel and M. Khovanov, Canonical bases in tensor products and graphical calculus for $U_{q}\left(\mathfrak{s l}_{2}\right)$, Duke Math. J., 87 (1997), 409-480.

[2] M. Kashiwara, Crystallizing the q-analogue of universal enveloping algebras, in 'Proceedings of the International Congress of Mathematicians', Kyoto, 1990.

[3] L.H. Kauffman and S.L. Lins, Temperley-Lieb recoupling theory and invariants of 3manifolds, Annals of Mathematics Studies, Princeton University Press, Princeton, NJ, 1994. 
[4] M. Khovanov, Graphical calculus, canonical bases, and Kazhdan-Lusztig theory, Ph.D. thesis, Yale University, 1997.

[5] G. Kuperberg, Spiders for rank 2 Lie algebras, Comm. Math Phys., 180(1) (1996), 109-151; q-alg/9712003.

[6] G. Lusztig, Introduction to Quantum Groups, Birkhäuser, Boston, 1993.

[7] G. Masbaum and P. Vogel, 3-valent graphs and the Kauffman bracket, Pacific J. Math., 164 (1994), 361-381.

[8] N.Yu. Reshetikhin and V.G. Turaev. Ribbon graphs and their invariants derived from quantum groups, Comm. Math. Phys., 127 (1990), 1-26.

Received April 28, 1997. The first author was supported by a Sloan graduate fellowship in mathematics. The second author was partially supported by NSF grant \#9423300.

University of CAlifornia, Davis

DAVIS, CA 95616

E-mail address: greg@math.ucdavis.edu 